\title{
Kidney Injury Associated with Cocaine Use
}

\author{
María Victoria Cabrera ${ }^{1}$, José Lucas Daza ${ }^{1 *}$, Gerardo Gutierrez ${ }^{1}$, Yaroslad de la Cruz ${ }^{1}$, Luis \\ Puello $^{1}$, Hernán Sarzuri ${ }^{1}$, John Galindo ${ }^{2}$, Abel Caraballo ${ }^{3}$, Néstor Guarnizo ${ }^{4}$ and Daniel Soto ${ }^{5}$
}

${ }^{1}$ Internal Medicine, Nephrology, University Buenos Aires, Argentina

${ }^{2}$ Nephrology unit, Fresenius Medical Care, Ibague, Colombia

${ }^{3}$ Intensivist Physician, Barranquilla, Colombia

${ }^{4}$ Intensive care unit, Tolima University, Colombia

${ }^{5}$ Manizales University, Colombia

*Corresponding author: José Lucas Daza, Internal Medicine, Nephrology, University Buenos Aires, Argentina

\begin{tabular}{ll} 
ARTICLE INFO & ABSTRACT \\
$\begin{array}{l}\text { Received: } \\
\text { Published: February 02, } 2022\end{array} \quad \begin{array}{l}\text { Cocaine consumption has multi-systemic consequences such as renal insufficiency, } \\
\text { hepatotoxicity, and lung toxicity. Acute and chronic renal changes are induced by } \\
\text { consumption. This study aimed at reporting two cases of acute renal damage with } \\
\text { different diagnoses related to cocaine use. }\end{array}$ \\
\hline
\end{tabular}

Citation: María Victoria Cabrera, José Keywords: Acute Kidney Injury; Cocaine Toxicity Lucas Daza, Gerardo Gutierrez, Yaroslad de la Cruz, Luis Puello, et al., Kidney Injury Associated with Cocaine Use. Biomed J Sci \& Tech Res 42(1)-2022. BJSTR. MS.ID.006689.

\section{Introduction}

Cocaine is a drug of abuse, currently considered a public health problem worldwide because of its addiction due to its use for recreational purposes. By 2019, around 20 million people between 15 - 45 years old ( $0.4 \%$ of the world population) have been cocaine users, however, due to its high demand, the trend continues to increase despite the availability of new alternatives to recreational drugs of abuse of synthetic origin [1]. After the isolation of coca paste by the German doctor Albert Nieman in 1865, the interest in this substance in the medicinal field increased, being mainly used as a local anesthetic in ocular surgery due to its vasoconstrictive properties; which led to a plethora of uses in medicine. Sigmund Freud extensively described its psycho-stimulant properties, and he used it as a treatment for some psychiatric disorders in particular those related to heroin abuse. Subsequently, recreational use popularized, particularly in the United States and Europe, the first cases of complications due to its massive consumption began to be reported in the 1980s. In 1982, it was reported the first myocardial infarction that was associated directly with cocaine 2 consumption [2]. The effects at the systemic level are widely documented [3], at low or moderate doses in the central nervous system it causes an increased mood, reduces appetite, causes insomnia, improves attention, and produces a surge in motor activity; in the cardiovascular system, it acts as a class I antiarrhythmic (at lower doses) by blocking sodium and potassium channels with a cardiodepressant and proarrhythmic effect, at higher doses; it stimulates endothelin 1 release, with a marked vasoconstrictive effect and it inhibits nitric dioxide production, causing hypertension and organ ischemia. 
A prothrombotic effect is recognized by increasing platelet aggregation and adhesion and reducing the activity of prostacyclin E2 [4]. It raises body temperature due to an increase in muscle activity, vasoconstrictive heat loss, and hypothalamic temperature control loss due to the depletion of dopamine receptors. All the systemic effects described are responsible for the complications on the different systems with deleterious effects both in the short and longterm [5] (Table 1). Specifically, renal involvement is often considered, although less frequently, mediated by rhabdomyolysis; nonetheless, it was also described that renal involvement was directly associated with vasculitis, direct tubulointerstitial injury, and glomerular vasoconstriction [6]. In this document we describe two clinical cases of acute kidney injury associated with cocaine use, we present their clinical characteristics and we will briefly review the literature on this topic.

Table 1: Systemic complications of cocaine.

\begin{tabular}{|c|c|}
\hline System & Complications \\
\hline Cardiovascular & $\begin{array}{c}\text { Arrhythmias, Myocardial Infarction, Myocarditis, } \\
\text { Endocarditis }\end{array}$ \\
\hline Neurological & Chronic Headache, Stroke, Vasculitis, Seizures \\
\hline Respiratory & $\begin{array}{c}\text { Sinusitis, Nasal Septum Perforation, Alveolar } \\
\text { Hemorrhage, Crack Lung Syndrome }\end{array}$ \\
\hline Muscular & Rhabdomyolysis, Myositis, Muscle Necrosis. \\
\hline Digestive & $\begin{array}{c}\text { Intestinal Perforation, Mesenteric Ischemia, } \\
\text { Pancreatitis, Hepatitis. }\end{array}$ \\
\hline Hematological & Thrombosis, Platelet Dysfunction. \\
\hline
\end{tabular}

\section{Case Report}

\section{Case 1: Clinical History}

30-year-old male patient, with no previous medical records. He consulted the emergency department on intense right lumbar pain of 48 hours of evolution without radiation, associated with macroscopic hematuria without a fever, chills, or dysuria. When questioned, he highlighted the use of inhaled cocaine for 2 years. On admission physical examination, vital signs are normal, afebrile, saturating 98 (0.21). with compression pain in the right flank. No signs of peritoneal irritation. Frankly positive right fist percussion. The rest of the physical examination did not show alterations.

Laboratory Tests Show: Hto: 40\% GB 11,800 (Ns 80\% L 20\%), normal urea and creatinine. Normal calcemia and uric acid, Total Bilirubin, Direct Bilirubin, FAL, TGP and TGO all of them at normal levels. Elevated LDH (x 5 times its normal value).

Urinary Sediment Examination: A field covered with isomorphic red blood cells is observed. Normal chest X-ray and electrocardiogram. The renal ultrasound did not show uronephrosis, urethral stones, or bladder abnormalities. A computed tomography (CT) scan showed hypodense and triangular external base images of the right kidney without post-contrast enhancement, characteristic of renal infarction (Figure 1). The case is interpreted as an acute renal infarction with preserved renal function associated with cocaine use. Treatment consisted of supportive measures with abundant hydration and opioid-based analgesia for pain management.

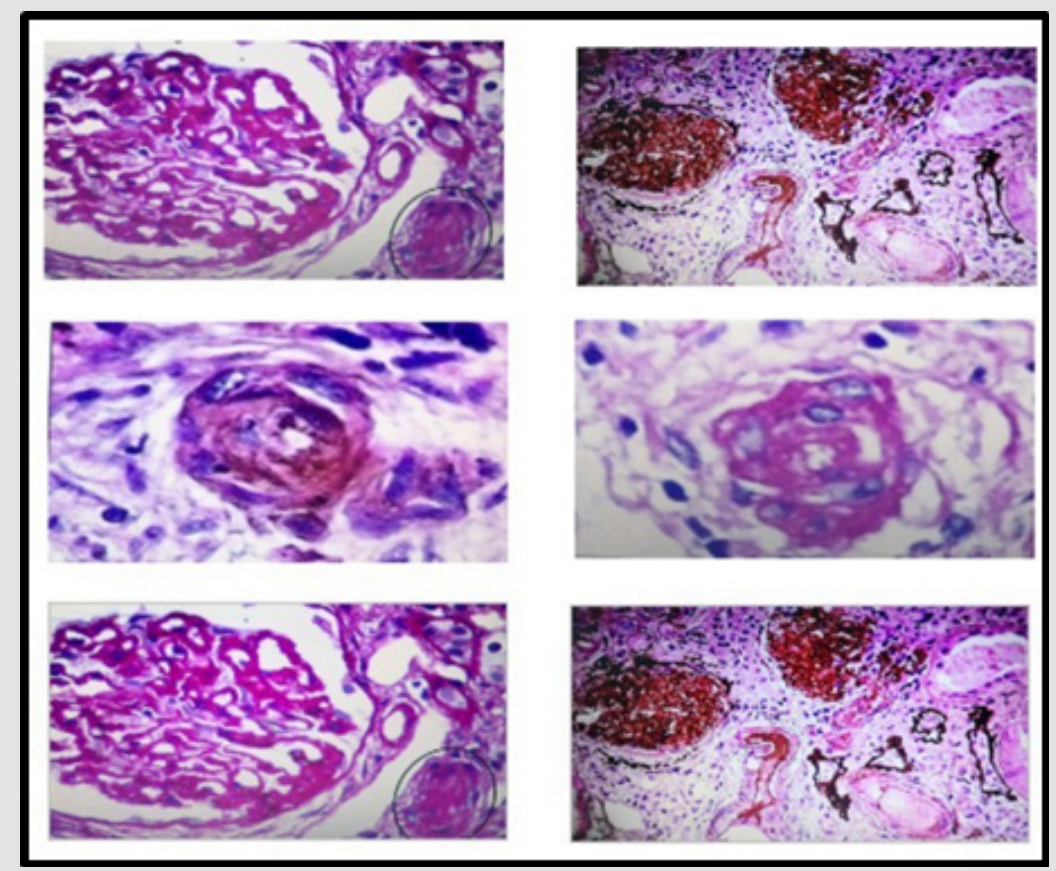

Figure 1: Renal biopsy images, performed by the Renal Pathology Service Hospital San Jose -Colombia, shows: thickening and wrinkling of capillary walls, arteries occluded by thrombus, concentric intimal hyperplasia, and onion-skinning. 
Evolution: The evolution was favorable, and hematuria resolved at 72 hours.

\section{Clinical Case 2: Clinical History}

40-year-old male patient of African descent diagnosed with hypertension. The medical records show that the previous 6 months he was in antihypertensive treatment, had severe alcoholism, and was a heavy smoker. He consulted the [2] emergency department for mild acute infection by COVID 19 and decompensated heart failure.

Laboratories: Hematocrit 35\%, hemoglobin: $12 \mathrm{~g} / \mathrm{dl}$, leukocytes: 9960, platelets: 219000, glucose: $122 \mathrm{mg} / \mathrm{dl}$, BUN 86 mg / dl, creatinine: $2.77 \mathrm{mg}$ / dl, sodium: $139 \mathrm{mEq}$ / L, potassium:3.7 mEq / L, LDH: 305 IU / L, D dimer: negative, serologies, ANCA, antiDNA, normal serum complement, negative viral serologies, normal thyroid profile. Toxicological tests are positive for the presence of cocaine in the blood.

Physical Examination: Arterial Tension: 200/110mmHg, heart rate. 87 beats per minute, respiratory rate 20 breaths per minute, temperature 36 degrees centigrade. Oriented in time, space and person; In the neck, it is observed jugular engorgement, crackles on bilateral pulmonary auscultation mainly in the bases, and lower extremity edema.

Electrocardiogram: In sinus rhythm with electrocardiographic data of left ventricular hypertrophy.

Renal Ultrasound: kidneys of preserved shape and size without alterations in the corticomedullary relationship.

Urinary Sediment: nondescript, without hematuria.

Evolution: He received supportive care, under monitoring in the intensive care unit, a negative fluid balance was induced with diuretics and later a coronary angiography was performed to evaluate possible coronary disease, which was reported normal. From the renal point of view, it was approached as an acute kidney injury AKIN II, with progressive renal function deterioration without anemia and without further data that suggests chronic kidney disease, consequently, a renal biopsy was performed.

Renal Biopsy Report: 25 glomeruli, 18 of them globally sclerosed, the remaining ones show ischemic signs of variable severity (capillary collapse, glomerular retraction, and pericapsular fibrosis), accompanied by podocyte alterations and in 2 of them, segmental sclerosis. The tubulointerstitial sector with pseudo thyroid classic atrophy (20\%), fibrosis (20\%), and focal mononuclear infiltrate. There are also groups of hypertrophied tubules with protein granules, cytoplasmic vacuoles, brush border loss, and isolated tubulitis. Arteries with moderate to severe intimal fibrosis, duplication of the elastic lamina, and presence of mucinous material in one of the branches. Arteries are mostly occluded by thrombi, concentric intimal hyperplasia, and/or sclerohyalinosis.

Immunofluorescence: $\operatorname{IgG}, \operatorname{IgA}$, and C1q: negative. IgM and C3 (2+) in accumulations, in areas of glomerular sclerosis, focal fibrinogen (+) in vascular walls, and Bowman's capsule.

Histopathological Diagnosis: Thrombotic Microangiopathy.

\section{Discussion}

Cocaine is known to affect all components of kidney tissue and the pathophysiological mechanisms involved in toxicity are multiple [6]. Cocaine-induced rhabdomyolysis caused by of acute renal failure was the first to be described in the medical records, the suggested mechanisms are ischemia, direct cellular injury due to increased production of oxygen free radicals production, vasoconstriction, and the myoglobin crystals produced by cocaine-induced muscle necrosis [7]. Some researchers reported ANCA-associated which might be related to the consumption of substances used to adulterate cocaine such as levamisole or alcohol used for cooking it (toxic metabolites) or those that increase the psychostimulant effect, which is a regular practice in recreational consumers [8]. Acute renal infarction is another mechanism described [9]. In the clinical case, 1 we describe the usual form of presentation of acute renal infarction with lumbar pain and hematuria, in a patient with a clear record of cocaine use, it could be the reason. There was no alteration of renal function, in this case, due to unilateral renal involvement. Anuria and complete renal failure with the requirement for renal support have been described in cases of bilateral renal involvement, however, it is an even rarer form of presentation [10]. right renal involvement agrees with that some researchers described and it is estimated that it is due to a bigger size of the renal artery and a multiplied resistance to flow, which might be related to microthrombus formation.

Data from observational studies and multiple case reports show the association of cocaine consumption with cardiac and cerebral thrombosis, suggesting that cocaine itself has a marked prothrombotic effect [11]. At the renal level, the mechanisms of the genesis of the renal infarction described are based on observational studies and animal models that seem to involve a combination of marked vasoconstriction due to an increase in circulating catecholamines, elevated levels of endothelin 1, and micro thrombosis due to alteration of platelet aggregation and an increase in thromboxane synthesis as well as alteration of intrarenal hemodynamics by a mechanism that is not clear $[10,11]$. There is no established treatment for renal thrombosis associated with cocaine use and in the clinical case that we describe, since there was no alteration in renal function, its evolution was favorable only with established support measures. In Clinical case number 2 , we describe a middle-aged man with a record of severe 
[2] polymedicated hypertension, acute kidney injury of unknown etiology, we performed a kidney biopsy that describes findings related to thrombotic microangiopathy.

A positive result in the test for alkaloids together with a finding of elevated LDH and other demographic data of the subject points to the possibility of its relationship with the surreptitious use of cocaine after ruling out other potential causes. The Thrombotic microangiopathy was seldom considered a cause of acute [0] renal failure and accelerated hypertension in cocaine users, the mechanisms are not fully recognized, they could be related to the combination of platelet microthrombus formation and direct endothelial damage due to severe hypertension that characteristic of this condition $[11,12]$. No immunity alterations were described in cocaine use, in contrast with other [0] secondary causes of drug- related thrombotic microangiopathy Although the prevalence of MIT is higher in women, the descriptions made in cocaine users place it predominantly in men, due to its higher consumption in this age group [13] (Diagram 1). Regarding the histological findings reported in our study, it can be verified that they belong to those usually described in thrombotic microangiopathies with renal involvement (notorious fibrinoid necrosis of the capillary tufts and glomerular arterioles) and that they reflect the renal tissue response to injury due to ischemia; [14] however, although the pathological findings mentioned in the recently described COVID 19 infection are multiple and affect all components of the renal tissue, the latter is associated with a predominantly tubulointerstitial and glomerular inflammatory involvement that is less common in patients with secondary thrombotic microangiopathies.

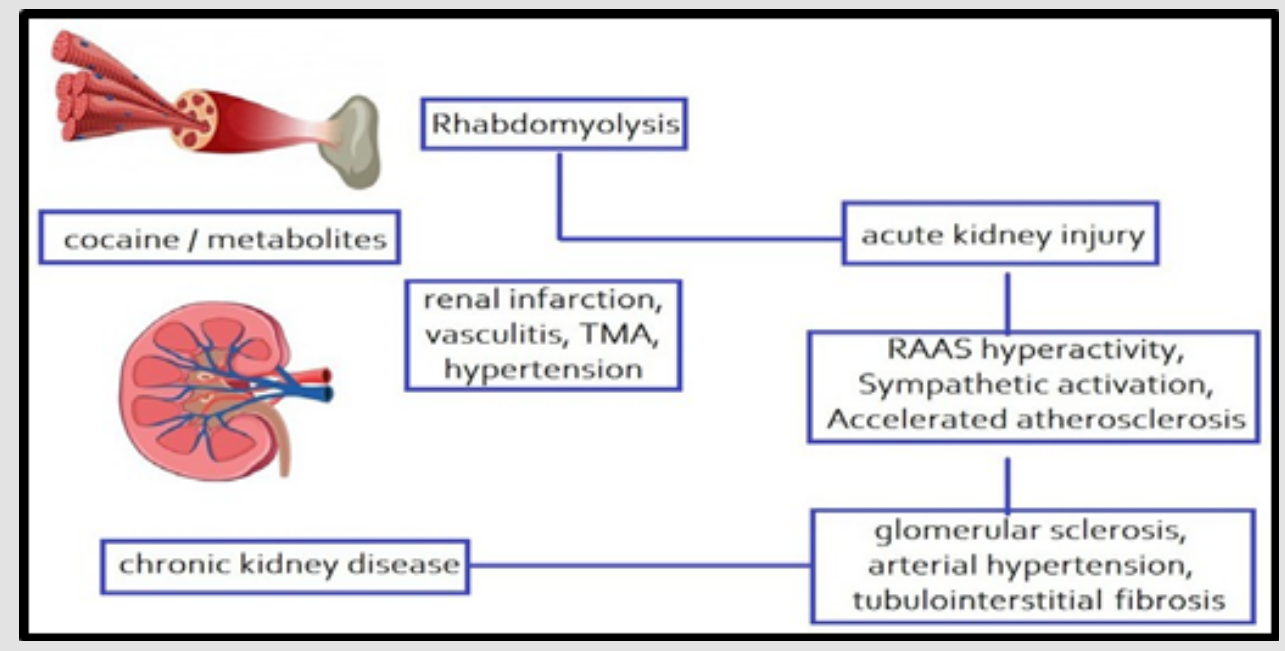

Diagram 1: Proposed pathophysiological mechanisms of renal injury induced by cocaine and/or its metabolites. MAT: Thrombotic Microangiopathy; RAAS: Renin-Angiotensin- Aldosterone System. Cocaine causes acute kidney injury by two mechanisms:

1) Indirectly After muscle injury, rhabdomyolysis occurs (the most frequent mechanism) and

2) Directly: Cocaine or its metabolites could induce direct injury by vasculitis, micro thrombosis formation, renal infarction, hypertensive nephropathy. Repeated episodes of acute kidney injury by mechanisms previously described would lead to the development of chronic kidney disease.

Some authors have reported viral inclusions in podocyte cytoplasm -not documented by the renal pathology group that evaluated the specimen in our description-, which reduces the prospects of a possible exclusively viral cause resulting in kidney damage without ruling it out completely [15]. Malignant hypertension as a mechanism of kidney injury associated with cocaine use has been supported by some authors in animal studies. Chronic cocaine use is [0] currently considered an independent risk factor for accelerated atherogenesis, which induces an increase in renin-angiotensin-aldosterone system activity, conditions hypertensive nephropathy [16] development. Although cocaine has not been directly established as a cause of chronic kidney disease, it is speculated that its chronic use is associated with repeated episodes of acute kidney injury together with malignant hypertension, they cause hemodynamic and structural changes due to oxidative stress, hypertension. Chronic disease mediated by vasoconstriction increased renin- angiotensin system activity, and accelerated atherogenesis causes increased mesangial matrix and tubulointerstitial fibrosis $[17,18]$. All of these conditions predispose to the development of chronic kidney disease.

\section{Conclusion}

Two cases are reported, the first with renal infarction, one of the rarest manifestations of cocaine-induced kidney damage 
without acute renal failure. The second case with cocaine- induced acute kidney injury without rhabdomyolysis or ischemic or toxininduced acute tubular necrosis. Renal failure with malignant hypertension was the main clinical manifestation in this patient. The histological characteristics were compatible with thrombotic microangiopathy. The mechanisms responsible for these pathological changes are unclear but are most likely multifactorial. Diffuse endothelial vascular injury due to direct toxicity or cocaineenhanced catecholamine release is likely a major contributor factor to the renal pathology observed in the 2 reported cases. Due to the diversity in the pathophysiological mechanisms involved in kidney damage, the final diagnosis usually requires a histopathological study; however, the importance of a comprehensive approach is highlighted, considering the possibilities in the differential diagnosis of acute kidney injury, particularly in young patients.

\section{References}

1. (2021) World Drug Report. United Nations publication, Sales No. E.21. XI.8.

2. National Library of Medicine.

3. Balcells, Mercedes (2001) Medical complications induced by cocaine. Adicciones 13: 167-177.

4. Lizasoain I Moro, MA Lorenzo P (2002) Cocaine: carmacological aspects. Addicciones 14(1): 57-64.

5. Quaglio G, Lugoboni F, Pajusco B, Fornasiero A, Mezzelani P, et al. (2004) Manifestazioni cliniche associate all'uso di cocaina. Ann Ital Med Int 19(4): 291-301.

6. Kanaan Mansoor, Murad Kheetan, Saba Shahnawaz, Anna P (2017) Shapiro. Systematic review of nephrotoxicity of drugs of abuse, 20052016. BMC Nephrology 18(1): 379.

ISSN: 2574-1241

DOI: $10.26717 / B J S T R .2022 .42 .006689$

José Lucas Daza. Biomed J Sci \& Tech Res

(C) (i) This work is licensed under Creative Commons Attribution 4.0 License

Submission Link: https://biomedres.us/submit-manuscript.php
7. Fokko J, van der Woude (2000) Cocaine use and kidney damage. Nphrol Dial Transaplan 15(3): 299-301.

8. Álvarez Díaz H, Marińo Callejo AI, García Rodríguez JF, Rodríguez Pazos L, Gómez Buela I, et al. (2013) ANCA-positive vasculitis induced by levamisole-adulterated cocaine and nephrotic syndrome: The kidney as an unusual target. Am J Case Rep 14: 557-561.

9. Wright NM, Martin M, Goff T, John Morgan, Rebecca Elworthy, et al (2007) Cocaine and thrombosis: a narrative systematic review of clinical and in-vivo studies. Subst Abuse Treat Prev Policy 2: 27.

10. Madhrira MM, Mohan S, Markowitz GS, Pogue VA, Cheng JT (2009) Acute bilateral renal infarction secondary to cocaine-induced vasospasm. Kidney Int 76(5): 576-580.

11. Filho JCCL, Ogawa MY, de Souza Andrade TH, Sami de Andrade Cordeiro Gadelha, Elizabeth De Francesco Daher, et al. (2019) Spectrum of acute kidney injury associated with cocaine use: report of three cases. BMC Nephrol 20: 99.

12. Goel N, Pullman JM, Coco M (2014) Cocaine, and kidney injury: a kaleidoscope of pathology. Clin Kidney J 7(6): 513-517.

13. Gu X, Herrera GA (2007) Thrombotic microangiopathy in cocaine abuseassociated malignant hypertension: report of 2 cases with review of the literature. Arch Pathol Lab Med 131(12): 1817-1820.

14. Brockleblank Vicky, Wood M Katrina, Kavanagh David (2018) Thrombotic microangiopathy and the kidney. Clin J Am Soc Nephrol 13(2): 300-317.

15. Sharma P, Ng JH, Bijol V, Jhaveri KD, Wanchoo R (2021) Pathology of COVID-19-associated acute kidney injury. Clin Kidney J 14(Suppl 1): i30-i39.

16. Brecklin CS, Gopaniuk Folga A, Kravetz T, Siama Sabah, Ashok Singh, et al. (1998) Prevalence of hypertension in chronic cocaine users. Am J Hypertens 11(11 Pt 1): 1279-1283.

17.Van der Woude FJ, Waldherr R (1999) Severe renal arterioarteriolosclerosis after cocaine use. Nephrol Dial Transplant 14(2): 434435 .

18. Sanchez PM, Perez CM, Romero M, Lorman R (2010) Cocaine use, high Blood Presure and Chonic kidney disease. Nefrologia 30(6): 599-714.

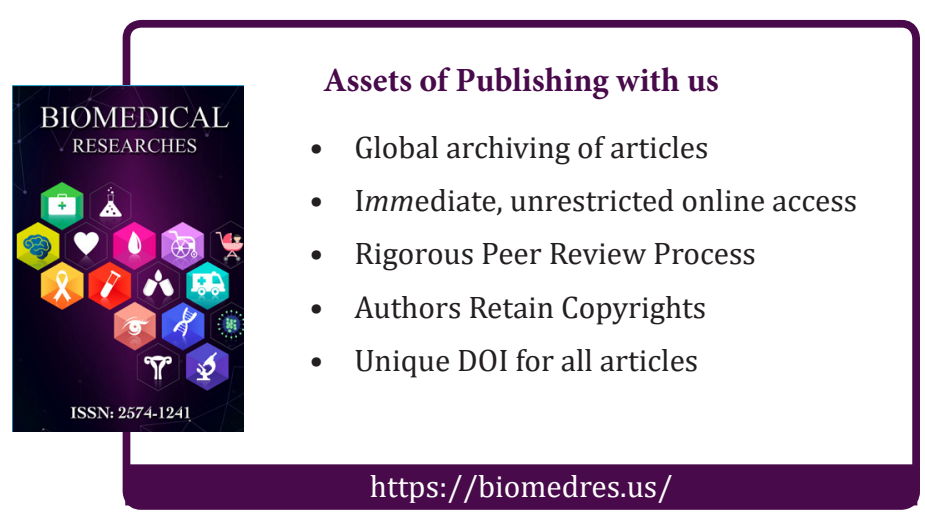

